\title{
Acknowledgement to Reviewers of Symmetry in 2017
}

\author{
Symmetry Editorial Office
}

MDPI AG, St. Alban-Anlage 66, 4052 Basel, Switzerland

Published: 12 January 2018

Peer review is an essential part in the publication process, ensuring that Symmetry maintains high quality standards for its published papers. In 2017, a total of 328 papers were published in the journal. Thanks to the cooperation of our reviewers, the median time to first decision was 19 days and the median time to publication was 42 days. The editors would like to express their sincere gratitude to the following reviewers for their time and dedication in 2017:

\author{
Abainia, Kheireddine \\ Abaza, Ayman \\ Abbas, Sherif \\ Achour, Hussam \\ Agell, Núria \\ Agliari, Elena \\ Ahmad, Fayyaz \\ Ahn, Sun Shin \\ Akavia, Adi \\ Akhtar, Zahid \\ Akram, Muhammad \\ Alam, Perwez \\ Alberola, Juan M. \\ Alcantud, José Carlos R. \\ Alizadeh, Mojtaba \\ Almeida, Joao Emilio \\ Almenárez, Florina \\ Alonso Jordá, Pedro \\ Alvarez, Rafael \\ Álvarez Rodríguez, José María \\ Amirtharajan, Rengarajan \\ Amiruzzaman, Md \\ Amza, Catalin \\ Anco, Stephen \\ Andreopoulou, Zacharoula \\ Antonio Jesús, Calderón Martín \\ Antonopoulos, Angelos \\ Antuchevičienè, Jurgita \\ Apiecionek, Łukasz \\ Archer, Gregory S. \\ Argia, Katsuhiko \\ Armour, John \\ Arrigo, Danny \\ Artese, Giuseppe \\ Arvanitis, Konstantinos \\ Arzano, Michele
}

\author{
Assante, Dario \\ Astrain, José Javier \\ Avarvari, Narcis \\ Awad, Ali Ismail \\ Babiarz, A. \\ Bade, Dirk \\ Bagdziunas, Gintautas \\ Baharon, Mohd Rizuan \\ Baiocchi, Valerio \\ Bairán, Jesús Miguel \\ Baker, Thar \\ Balagué, Natàlia \\ Baležentis, Tomas \\ Bamba, Kazuharu \\ Ban, Adrian I. \\ Banić, Nikola \\ Bao, Yue \\ Bardakov, Valeriн \\ Barton, Michael \\ Baule, Adrian \\ Bausys, Romualdas \\ Becattini, Niccolò \\ Behura, Sanjay \\ Bein, Wolfgang \\ Belhadj Amor, Selma \\ Bender, Paul \\ Ben-Romdhane, Hajer \\ Berezansky, Leonid \\ Besieris, Ioannis M \\ Bezdek, Karoly \\ Bhotto, Md. Zulfiquar Ali \\ Bianucci, Marco \\ Bielinis, Ernest Paweł \\ Bila, Nicoleta \\ Bithas, Petros S. \\ Blanc, Sara
}


Bobulski, Janusz

Boccuto, Antonio

Bohr, Jakob

Bonati, Beatrice

Bonezzi, Roberto

Bonyadi, Mohammad Reza

Borkowski, Piotr

Botta, Marco

Bouganis, Christos-Savvas

Bozalakov, Dimitar

Brandt, Steven R.

Bremer, Jörg

Brown, Stephen

Brownlee, Sandy

Bukkapatnam, Satish T.S.

Burdescu, Dumitru

Bustince, Humberto

Cabrerizo, Francisco Javier

Caleffi, Marcello

Calvo, Paula

Campbell, Julie

Campbell, Abraham

Campoamor Stursberg, Rutwig

Cantrell, Gary

Cao, Siyang

Cao, Xin

Caprara, Sergio

Caramés, Tiago Fernández

Carbone, Paolo

Carcaud, Julie

Carlini, Maurizio

Carminati, Maria Chiara

Carrillo De Gea, Juan Manuel

Casaca, Wallace

Castaldi, Giuseppe

Casteren, Jan Van

Castle, Toen

Catalano, Domenico

Cattani, Carlo

Cattelani, Luca

Cavagnino, Davide

Cazabet, Remy

Čereška, Audrius

Cerny, Tomas

Cerreta, Maria

Chadli, Mohammed

Chakeri, Alireza

Chan, Yu-Wei

Chan, Keith C. C.

Chang, Wan-Jung

Chao, Liu

Charalampos, Pitas
Chargé, Pascal

Chaudy, Yaëlle

Chellamuthu, Vinodh

Chen, Chien-Chung

Chen, Chi-Hua

Chen, Shyi-Ming

Chen, Yulin

Chen, Bo

Cheng, Chin-hsing

Cheng, Tsang-Hsiang

Cheng, Nan

Cherniha, Roman M.

Chessa, Manuela

Chiacchio, Ferdinando

Chiandetti, Cinzia

Chiarelli, Piero

Chiementin, Xavier

Chih, Mingchang

Chmielewska, Aleksandra

Choi, Wookjin

Choi, Bong Jun

Choi, Sangil

Choo, Kim-Kwang

Chou, Jue-Sam

Chu, Shu-Chuan

Chung, Chun-Jen

Chung, Yao-Liang

Chung, Tein-Yaw

Ciecholewski, Marcin

Ciliberto, Sergio

Cimato, Stelvio

CIOCA, Lucian-Ionel

Ciuonzo, Domenico

Coleman, Anthony W.

Collins, John James

Collins, Maurice N.

Concezzi, Moreno

Conejero, J. Alberto

Coors, Volker

Corballis, Michael

Corda, Christian

Cordier, Frédéric

Cortes, Jose

Costa-Requena, Jose

Couchot, Jean-François

Craus, Mitică

Crippa, Paolo

Crocco, Lorenzo

Cuadra, Lucas

Cuello, Luis G.

Curiac, Daniel-Ioan

Da, Fang 
Da Fonseca, Carlos

Dąbrowski, Paweł

Dala, Laurent

Dalhuisen, Jan Willem

Dalton, Lori

Damaševičius, Robertas

D'Andreagiovanni, Fabio

Dassios, Ioannis K.

Davidrajuh, Reggie

Davidsson, Paul

Davydovych, Vasyl'

De, Suparna

De Andrés Calle, Rocío

De Diego, Isaac Martín

De La Cruz Dombriz, Álvaro

De Meo, Pasquale

Debono, Ivan

Dehmer, Matthias

Demestichas, Konstantinos

Demir, Mustafa

Deng, Yong

Desai, Alok

Di Crescenzo, Antonio

Di Martino, Ferdinando

Diez, Matteo

Ding, Jintai

Dixon, David A.

Dobrescu, Bogdan

Dolgushev, Maxim

Dombi, József

Doria, Alberto

Dorst, Leo

Dosen, Strahinja

Dragoi, Marian

Dragon, Norbert

Dreżewski, Rafał

Droghei, Riccardo

Du, Wei-Shih

Dudczyk, Janusz

Duggal, Krishan

Dulebenets, Maxim A.

Duplij, Steven

Dziurdzia, Piotr

Egon, Schulte

Eldawy, Ahmed

Eliasson, Jens

Elinder, Fredrik

Ellison, Nick

Ellzey, M. Lawrence

Elsts, Atis

Emmerich, Michael

Esposito, Antonio
Esposito, Christian

Evans, Michael

Evsutin, Oleg

Fabri, Mara

Fadda, Mauro

Fang, Shih-Hau

Favini, Angelo

Fedorchuk, Vasyl

Fehribach, Joseph

Feldman, Alexander

Feng, Yunlong

Fenza, Giuseppe

Fernandes Jr., Gilberto

Fernandez Fernandez, Luis Manuel

Ferrara, Massimiliano

Ferreira, Milton

Figlus, Tomasz

Filho, Edson

Finck, Steffen

Fink, Olga

Fiore, Ugo

Fischer, Jean-Paul

Fliegel, Karel

Fortuna, Luigi

Fowler, Michael

Fraga-Lamas, Paula

Francisco José, Sánchez Sutil

Francotte, Eric

Franklin, Manoj

Frasnelli, Elisa

Frassino, Antonia M.

Frau, Juan

Friedenberg, Jay

Froelich, Wojciech

Frontoni, Emanuele

Fryazinov, Oleg

Fujii, Keisuke

Fujii, Hidemichi

Fullér, Robert

Furtula, Boris

Fuster-Parra, Pilar

Gabriel, Jose Luis

Gadouleau, Maximilien

Gain, James

Gamo, Javier

Gan, Guojun

Gao, Xiaolong

Gao, Longxiang

Garcia, Jordi

García, Carmelo R.

García Díaz, Pilar

Garcia-Hernandez, Laura 
Garcia-Pedrero, Angel

Garcia-Valls, Marisol

Gargano, Francesco

Gasulla, Manuel

Gay, Michel

Geelan, David

Gendron, Paul

Geng, Ruili

Gessner, Guido

Gheorghe, Grigoras

Gheribi, Aimen.E.

Giambo', Roberto

Giannakis, Konstantinos

Giannelli, Carlo

Gil, David

Ginters, Egils

Gkountaroulis, Dimoklis

Glowacz, Adam

Godang, Romulus

Godinez Tello, Richard

Gogolin, Greg

Golob, Marjan

Gomez, Luis

Gong, Wenping

Goodrich, Christopher S.

Gope, Prosanta

Górka, Jakub

Gostar, Amirali Khodadadian

Goswami, Prashant

Goubko, Mikhail

Gramaglia, Marco

Grammenos, Theofanis

Green, David

Grimmer, Hans

Grøn, Øyvind

Groote, Stefan

Grossman, Jerrold W.

$\mathrm{Gu}$, Fengshou

Guendelman, Eduardo

Guichard, Paul

Güntürkün, Onur

Guo, Yanhui

Gutiérrez, Eduardo Quevedo

Guttmann, Anthony

Hadley, Mark J.

Haklev, Stian

Hallgrimsson, Benedikt

Hamza, Ben

Han, Seog-young

Hao, Fei

Harders, Matthias

Harish, Garg
Harko, Tiberiu

Hashemi, Morteza

Hashizume, Hiromichi

Hatzopoulos, Georgios

Heinimann, Hans

Helseth, Lars

Henesey, Lawrence

Hepworth, Ammon

Herbert, Andrew M.

Hernández-Gómez, Juan Carlos

Herrera, Raquel

Herrera-Viedma, E.

Herrero, Rolando

Heuzé, Yann

Hidalgo, Arturo

Hikida, Yasuaki

Hildenbrand, Dietmar

Hofmann, Ralf

Hong, Youn-Sik

Hong, Wien

Hong, Daesik

Hong, Wei-Chiang

Hoover, Kara C.

Hortal Quesada, Enrique

Hou, Kun-mean

Hristova, Snezhana

Hsieh, Chiu-Fan

Hsieh, Jun-Wei

Hsu, Ming-wei

$\mathrm{Hsu}$, Ming-Fu

Hsu, Li-Yi

Huang, Yishuo

Huang, Jiangshuai

Huang, Jenny

Huang, Chester S. J.

Huang, Xiaodi

Huang, Guan-Shieng

Humphries, Stephen

Hurd, Peter L.

Iancu, Ion

Iantovics, Laszlo Barna

Ibl, Martin

Ieong, Ricci

Ikai, Tomoyuki

Ilić, Aleksandar

Ilmonen, Pauliina

Invitto, Sara

Iodice, Paolo

Iorio, Lorenzo

Ishida, Haruma

Islam, Syed

Ivanov, Yury 
Iyad, Lahsen Cherif

Jaatun, Martin

Jaba, Elisabeta

Jacobs, George

Jacobsen, Elisabeth Egholm

Jacyna, Marianna

Jakóbczak, Dariusz Jacek

Jalal, Ahmad

Jamdagni, Aruna

Janc, Krzysztof

Jasra, Ajay

Javaid, Ahmad Y.

Jayaraman, Prem

Jaycay, Robert

Ji, Zhanlin

Jiang, Fan

Jiménez-Martín, Antonio

Jin, Kyong Hwan

Jin, Yan

Jirgl, Miroslav

Jonckheere, Edmond

Jones, Alex

Jong, Gwo-jia

Joo, Sung Jun

Jorge, Paulo Esteves

Jose Ignacio, Rojas-Sola

Jothi, Sathiskumar

Jung, Ki-Hyun

Jurman, Giuseppe

Kaabouch, Naima

Kaklauskas, Artūras

Kaminski, Marcin

Kamisalic, Aida

Kamsu-Foguem, Bernard

Kanamori, Takafumi

Kanavos, Andreas

Kandris, Dionisis

Kang, H-Y

Kannan, Ravi

Kantarci, Burak

Kapociute-Dzikiene, Jurgita

Karenina, Karina

Karthikeyan, P.

Karypis, George

Kashino, Kunio

Katarzyna, Rudnik

Kaucic, Massimiliano

Kauffman, Louis H.

Kazimieras Zavadskas, Edmundas

Ker, Jun-ing

Keršulienè, Violeta

Khalaf, Walaa
Khan, Saad

Khan, Usman $\mathrm{T}$

Kharzeev, Dmitri E.

Khlopov, Maxim Yu.

Khoddamzadeh, Amir

Kiang, Jean-Fu

Kim, Seong Dae

KIM, Cheonshik

Kim, Hyunsung

Kim, Chang Hun

Kim, Donghoon

Kim, TaiHoon

Kim, Cheonshik

Kleidis, Kostas

Klement, Erich Peter

Klimach, Harald

Klumpp, Matthias

Ko, Chia-Nan

Koch, Eckhard

Kołakowska, Agata

Kondylakis, Haridimos

Kongar, Elif

Kooijman, J. D. G.

Korbel, Jan

Koskenoja, Mika

Kotsiantis, Sotiris

Kotter, Richard

Koumoulis, Dimitrios

Koutromanos, Georgios

Král, Pavel

Krämer, Manuel

Kratzke, Nane

Kreinovich, Vladik

Krejcar, Ondrej

Krüger, Björn

Kruse, Rudolf

Kučerovský, Dan

Kulshreshth, Arun

Kumamoto, Kazuo

Kuremoto, Takashi

Kurti, Arianit

Kuzawa, Chris

Kwak, Keun-Chang

Kwok, Tsz-Ho

La Scalia, Giada

Lalande, Jean-Francois

Lamberti, Luciano

Lameiro, Christian

Land, Martin

Landrieu, Loic

Lean, Madeleine Jotz

Leccese, Fabio 
Lecomte, Claude

Lecroq, Thierry

Lee, Jae Woong

Lee, YoungDoo

Lee, Cheng-Ming

Lee, Tian-Fu

Lee, Da-Sheng

Lee, Chul Ung

Lee, Sang Hyun

Lee, Taekkyeun

Lee, Shyi-Long

Leibovici, Didier

Leijon, Arne

Lemanska, Magda

Leonowicz, Zbigniew

Leopold, Undine

Li, Changzhi

Li, Yung-Hui

Li, Rongjian

Li, Peter

Li, Kezhi

Li, Tiancheng

Lian, Wenzhao

Liang, Feng

Liang, Yu

Lin, Tyrone $\mathrm{T}$.

Lin, Junlin

Lin, Feng-Cheng

Lin, Chien-Chou

Lin, Chi-Ying

Lin, Chun-Ling

Listanti, Marco

Liu, Wen-Chen

Liu, Zilei

Liu, Jain-Shing

Liu, Qiang

Liu, Ran

Liu, Peide

Liu, Shing-Hong

Liu, Ziping

Lombardi, Flavio

Lomenie, Nicolas

Longo, Sandro

Lopes, Nuno

Lopez De Lacalle, Oier

Lopez-Pellicer, Francisco J.

Losee, Robert M.

Lotfi, Ahmad

Lu, Tzu Chuen

Lu, Rongxing

Luan, Vu Thai

Lubchenko, Vassiliy
Luneau, Dominique

Luo, Jia-Ning

Luongo, Orlando

$\mathrm{Ma}$, Wenxiu

Ma, Wen Xiu

Macchi, Piero

Machado, Tenreiro

Madej, M. Gregor

Mahabir, Ron

Majewski, Maciej

Makin, Alexis

Makris, Christos

Maksymyuk, Taras

Malashichev, Yegor

Malomed, Boris

Man, Ka Lok

Manev, Nikolay

Mangazeev, Vladimir V

Manning, John

Manns, Martina

Manteaux, Pierre-Luc

Marciniak, Marcin

Marcolin, Federica

Mariani, Camillo

Maros, Katalin

Marrone, Stefano

Marszałek, Zbigniew

Martalò, Marco

Martinez, Luis

Martinez-Izquierdo, Estibaliz

Martins, Luísa

Martins-Ferreira, Nelson

Masini, Barbara Mavi

Masse, Antoine

Mathes, D. Benjamin

Maximiano, Marisa Da Silva

Mazumdar, Somnath

McAlister, Ruth

McClean, Sally

McShane, Blakeley

Meisen, Tobias

Melvin, William L.

Mendes, Jérôme

Meng, Weizhi

Merigo, Jose M.

Meseguer, Roc

Mezei, József

Michailidis, Panagiotis

Michalski, Radosław

Milej, Daniel

Milton, Kimball

Miñana, Juan-José 
Mirjalili, Seyedali

Mitarbeiterin, Wissenschaftliche

Mityushev, Vladimir

Miyazaki, Shuichi

Mkwawa, Is-Haka

Mls, Karel

Moazzemi, Reza

Moh, Sangman

Mokhtari, Ghassem

Møller, Anders

Monaco, John V.

Mondal, Soumik

Montero, Francisco Javier

Moreno Pineda, Eufemio

Morimitsu, Henrique

Morisi, Stefano

Mormann, Thomas

Moroni, Davide

Morozs, Nils

Mortimer, Michael

Moshou, Dimitrios

Motta, Gianmario

Mould, David

Mowshowitz, Abbe

Moylan, Patrick

Mundani, Ralf-Peter

Murinova, Petra

Musialski, Przemyslaw

Musslimani, Ziad

Naghshin, Vahid

Naik, Ganesh

Nalepa, Jakub

Namiot, Dmitry

Nandwana, Vikas

Nanni, Loris

Napoli, Christian

Naranjo, Jose Eugenio

Nasrollahi, Amir

Navara, Mirko

Navarro, Nicolas

Nazarko, Lukasz

Nekrasov, Alexey

Neto, Pedro

Neumann, Knut

Neumann, Peter M.

Nguyen, Ngoc Tu (Ryan)

Nieto, Juan J.

Nieto, Ana

Nino-Ruiz, Elias D.

Nobile, Marco S.

Nour-Eldin, Hussam Hassan

Nowak, Piotr
Nozick, Vincent

Nyka, Krzysztof

Ocklenburg, Sebastian

O'Connell, Kevin F.

Oikonomou, V.K.

Okarma, Krzysztof

Olaizola, Igor García

Oliveri, Francesco

Olivier, Stephen Lecler

Ori, Ottorino

Oriwoh, Edewede

O'Shaughnessy, Douglas

Oszust, Mariusz

Overgaard, Jacob

Pabbidi, Reddy M

Palafox, Leon

Paliathanasis, Andronikos

Pamučar, Dragan

Panagiotakis, Costas

Panwisawas, Chinnapat

Papadimitriou, Fivos

Papadopoulos, Basil K.

Papakiriakopoulos, Dimitris

Papakostas, George

Paradis, Kyle F

Paravastu, Narasimha

Paredes, Hugo

Parikesit, Arli Aditya

Park, Choonkil

Park, MyeongJin

Parlow, Shelley

Parrein, Benoît

Parvan, Alexandru S.

Pasini, Dario

Pastuszak, Grzegorz

Paternain, Daniel

Patriota, Alexandre Galvão

Pau, Giovanni

Paulus, Christoph J.

Pavelka, Michal

Pecorella, Tommaso

Pecori, Riccardo

Pelusi, Danilo

Penne, Rudi

Peppas, Kostas P.

Perboli, Guido

Perc, Matjaz

Perdigão, Rui A. P.

Pérez, Valentín

Perez-Alvarez, Ivan

Perkins, Andy D.

Petcu, Dana 
Peterson, Mary

Petit, Nicolas

Petraglia, Mariane R.

Petrellis, Nikos

Pettersson, William

Pilato, Giovanni

Pinoli, Jean-Charles

Pirk, Soeren

Pizzolante, Raffaele

Pławiak, Paweł

Pogány, Tibor K.

Polonyi, Janos

Pooranian, Zahra

Popelka, Stanislav

Popowicz, Ziemowit

Portilla, Ana

Pouke, Matti

Pranggono, Bernardi

Precup, Radu-Emil

Prentkovskis, Olegas

Profumo, Stefano

Przybyła-Kasperek, Małgorzata

Puchinger, Jakob

Putz, Mihai V.

Qian, Chuanxi

Qiao, Yuansong

Qin, Zhengrui

Qiu, Jianbin

Qu, Guangzhi

Radac, Mircea-Bogdan

Radenovic, Stojan

Radin, Michael

Rahbarinia, Babak

Rakhshan, Ali

Ramasamy, Subramanian

Ramaswamy, Palaniappan

Rasti, Pejman

Rastogi, Vaibhav

Ratava, Juho

Rathore, Saima

Rawassizadeh, Reza

Ree, Sangwook

Reina, Daniel Gutiérrez

Reis, Arsénio

Reiter, Clifford A.

Renzi, Polyssena

Requardt, Manfred

Rezaei, Jafar

Ribeiro-Soriano, Domingo Enrique

Riedstra, B.J.

Riera, Juan Vicente

Ripon, Kazi Shah Nawaz
Ritchie, Kay

Rizzardi, Alessandra

Rodger, James A.

Rodino, Luigi

Rodrigues, Alexandre A. P.

Rodriguez, Dionisio

Rodríguez, Rosa M.

Rosanna, Costaguta

Roussigné, Myriam

Roy, Swapnoneel

Rubi, Miguel

Rubin, Stuart H.

Rubio-Sánchez, Manuel

Ruotolo, Francesco

Russ, John

Sadun, Lorenzo A.

Saikawa, Kenichi

Sainz, Nekane Ione

Sakajo, Takashi

Sakalauskas, Virgilijus

Sakar, Biswajit

Sakilam, Satish

Sałabun, Wojciech

Samvedi, Avinash

Sang, Nguyen Quang

Sanganyado, Edmond

San-Segundo, Rubén

Santos, Jesús Daniel

Santosh, K.C.

Saridakis, Emmanuel

Sarlis, Prof. N

SATO, Hiroyuki

Satorra, Albert

Savriama, Yoland

Scannapieco, Antonio Fulvio

Schaffer, Henry E.

Schagaev, Igor

Schein, Stan

Schein, Stan

Schinas, C.J.

Schmitt, Andreas

Schmoecker, Jan-Dirk

Schoening, Timm

Schroeder, MARCIN

Schulz, Benjamin L.

Schumacher, Jens

Schurig, Volker

Schwab, Arend L.

Scioscia, Floriano

Sciriha, Irene

Sedgwick, Hal

Seer, Caroline 
Seitz, Jochen

Selva, Josue

Seo, Dong-Hoan

Serra-Capizzano, Stefano

Sessa, Salvatore

Shadloo, Mostafa

Shao, Lijing

Shao, Chenhui

Shao, Lijing

Sharman, Rebecca J

Sheng, Yunhe

Shi, Peng

Shiau, Tzay-An

Shojafar, Mohammad

Shon, Kwang

Shroff, Piyush

Sidiropoulos, Panagiotis

Sigarreta, José $\mathrm{M}$.

Sila-Nowicka, Katarzyna

Silva, Rui

Sinelshchikov, Dmitry

Singh, Pushpinder

Skilton, Mark

Skrzypczyński, Piotr

Sladkowski, Jan

Smarandache, Florentin

Sofiane, Guessasma

Song, Wei

Sookhak, Mehdi

Sostak, Alexander

Sousa, J.C.

Souto, André

Sparavigna, Amelia Carolina

Spjut, Josef B.

Sreerama, Subramanya

Staiano, Antonino

Stavrinos, Panayiotis

Stentoumis, Christos

Šter, Branko

Stetter, Ralf

Stoettinger, Elisabeth

Stone, Michael

Strother, Lars

Strowes, Stephen D.

Studer, Harald P.

$\mathrm{Su}$, Ruoyu

$\mathrm{Su}$, Ming-Shou

Suarez, Alvaro

Sugano, Masashi

Suh, Jangwon

Sukhov, Vladimir

Sung, Yunsick
Sung, Yunsick

Sun-Yuan, Hsieh

Szabo, Lorand

Szekely, Gyorgy

Tafuni, Angelantonio

Tahmasseby, Shahram

Targiel, Krzysztof

Tasson, Jay

Tawfik, Abdel Nasser

Tchórzewska-Cieślak, Barbara

Tegowski, Jaroslaw

Teh, Pin Shen

Teknomo, Kardi

Teper, Roee

Thakker, Dhaval

Theilmann, Florian

Theocharis, John B.

Thiery, Alex

Tichý, Tomáš

Tieri, Gaetano

Toivanen, Miika

Tominski, Christian

Toropov, Andrey A

Torrens, Francisco

Torres, Pedro M. B.

Torres-Sospedra, Joaquín

Tramontana, Emiliano

Tran, Le Nam

Triviño, Alicia

Trueba, José L.

Tsai, Yuan-Yu

Tsiigkaridis, John (Ioannis)

Tsinos, Christos G.

$\mathrm{Tu}$, Jih-Fu

$\mathrm{Tu}$, Michael

Turaev, Vladimir

Uddin, Shahadat

Ullah, Shatif

Ungurean, Ioan

Upadhyay, Anup

Uslar, Mathias

Uttam, Shikhar

Uva, Antonio

Vacaru, Sergiu I.

Valera, Alvin C.

Valero-Verdú, Sergio

Vallortigara, Giorgio

Van Der Helm, Peter A.

Van Dongen, Stefan

Van Enk, Steven J.

Vangelista, Lorenzo

Varevac, Damir 
Vaughan, Neil

Venckauskas, Algimantas

Venkatesan, Rajasekar

Verma, Sanny

Versace, Elisabetta

Vien, Quoc-Tuan

Vigneron, Antoine

Vîlcu, Gabriel-Eduard

Villani, Claudio

Visintin, Phillip

Vitale, Patrizia

Vladareanu, Luige

Vladutescu, Stefan

Volná, Eva

Vulpe, Alexandru

Vvedensky, Dimitri

Wade, T. Joel

Wang, Hua

Wang, Shaohui

Wang, Shuhang

Wang, Mengyu

Wang, Shaohui

Wang, Hai

Wang, Shu-ching

Wang, Dong

Wang, Shaohui

Wang, Jack

Wang, Yun

Watel, Dimitri

Wątróbski, Jarosław

Wei, Yufeng

Weitzen, Jay A.

Wells, Deborah

Wen, Chih-Yu

Wen, Kunli

Whitlock, Paula

Wibowo, Santoso

Wichardt, Philipp C.

Wicherts, Jelte

Wilder, John

Witt, Carsten

Wojciechowski, Adam

Wolf, Guy

Won, Myounggyu
Wong, Ka-Chun

Woźniak, Marcin

Wright, Michael

Wu, Weining

$\mathrm{Wu}$, Tzong-Chen

Wysocki, Marian

Xi, Peng

Xie, Jiafeng

$\mathrm{Xu}$, Hong-Kun

Yamato, Yoji

Yan, Mingyuan

Yang, Laurence T.

Yang, Yimin

Yang, Can

Yang, Tiantian

Yazdani, Morteza

Ye, Wenlei

Yen, Neil Y.

Yi, Gangman

Yin, Penghang

Yiu, Man Lung

Yoon, Dae Won

You, Shingchern

Youssofzadeh, Vahab

Yu, Weiren

Yu, Chunyang

Yu, Yang

Yuan, Zhongyi

Yuen, Chau

Zalyubovskiy, Vyacheslav

Zambelli, Cristian

Zanlungo, Francesco

Zavadskas, Edmundas Kazimieras

Zhang, Jie

Zhang, Xianming

Zhang, Yang

Zhang, Bowu

Zhang, Jiashu

Zhang, Bowu

Zhang, Tonghua

Zhou, Xiran

Zhou, MengChu

Zwanzig, Silvelyn

(C) 2018 by the authors. Licensee MDPI, Basel, Switzerland. This article is an open access article distributed under the terms and conditions of the Creative Commons Attribution (CC BY) license (http://creativecommons.org/licenses/by/4.0/). 